\title{
Efficacy of simultaneous aerobic exercise and cognitive training in subjective cognitive decline: study protocol for randomized controlled trial of the Exergames Study
}

\author{
Dereck Salisbury $^{1 *}$ (D) Tom Plocher ${ }^{2}$ and Fang $\mathrm{Yu}^{1,3^{*}}$
}

\begin{abstract}
Background: Subjective cognitive decline (SCD) is an early manifestation of Alzheimer's disease (AD) and offers a therapeutic window where interventions have strong potential to prevent or delay the progression of AD. Aerobic exercise and cognitive training represent two promising interventions for $A D$ prevention, but their synergistic effect has yet to be assessed in persons with SCD.

Methods/design: The purpose of this single-blinded, 3-parallel group randomized controlled trial is to test the synergistic efficacy of an exergame intervention (simultaneous moderate-intensity aerobic cycling and cognitive training) on cognition and aerobic fitness in community-dwelling older adults with SCD. The Exergames Study will randomize 96 participants on a 2:1:1 allocation ratio to 3-month exergame, cycling only, or attention control (stretching). Primary outcomes include global cognition and aerobic fitness, which will be assessed at baseline and after 3 months. The specific aims of the Exergames Study are to (1) determine the efficacy of the exergame in older adults with SCD and (2) assess the distraction effect of exergame on aerobic fitness. Data will be analyzed using ANOVA following intention-to-treat.
\end{abstract}

Discussion: This study will test the synergistic effects of exergame on cognition and aerobic fitness. It has the potential to advance prevention research for AD by providing effect-size estimates for future trials.

Trial registration: ClinicalTrials.gov NCT04311736. Registered on 17 March 2020.

Keywords: Aerobic exercise, Cognitive training, Cognition, Subjective cognitive decline, Exercise, Alzheimer's disease

\footnotetext{
*Correspondence: salis048@umn.edu; yuxxx244@umn.edu;

Fang.Yu.2@asu.edu

'University of Minnesota School of Nursing, 5-160 WDH 1331, 308 Harvard St

SE, Minneapolis, MN 55455, USA

Full list of author information is available at the end of the article
}

(c) The Author(s). 2021 Open Access This article is licensed under a Creative Commons Attribution 4.0 International License, which permits use, sharing, adaptation, distribution and reproduction in any medium or format, as long as you give appropriate credit to the original author(s) and the source, provide a link to the Creative Commons licence, and indicate if changes were made. The images or other third party material in this article are included in the article's Creative Commons licence, unless indicated otherwise in a credit line to the material. If material is not included in the article's Creative Commons licence and your intended use is not permitted by statutory regulation or exceeds the permitted use, you will need to obtain permission directly from the copyright holder. To view a copy of this licence, visit http://creativecommons.org/licenses/by/4.0/. The Creative Commons Public Domain Dedication waiver (http://creativecommons.org/publicdomain/zero/1.0/) applies to the data made available in this article, unless otherwise stated in a credit line to the data. 


\section{Background}

Subjective cognitive decline (SCD), the subjective experience of worsening memory or cognitive, is one of the earliest noticeable symptoms of Alzheimer's disease (AD) [1]. It offers a therapeutic window where interventions have strong potential to prevent or delay the progression of $\mathrm{AD}$ because of the increasing recognitions that AD pathology accumulates over years to decades before any observable clinical symptoms [2]. Interventions at later clinical phases of $\mathrm{AD}$ have been ineffective or shown only modest benefits [3]. Aerobic exercise and cognitive training are two potential disease-modifying interventions through the induction of brain plasticity and attenuation of AD neurodegeneration [4-6]. Epidemiological studies have linked exercise to reduced risk for AD [7-9]. Likewise, randomized controlled trials (RCT) and meta-analyses of RCTs demonstrate that aerobic exercise produced mild to moderate gains in a wide range of cognitive domains in persons with intact cognition, mild cognitive impairment (MCI), or dementia [10-15]. On the other hand, cognitive training involves repeated practice of a set of standard cognitive tasks targeting specific cognitive domains [16]. Targeting cognitive processes (e.g., attention) may produce more robust and broadly generalizable effects than targeting cognitive structures (e.g., delayed recall) $[17,18]$. Previous studies found that such process-based cognitive training generated a moderate to large improvement in selected cognitive domains in older adults with intact cognition or MCI [19-21].

Given that aerobic exercise and cognitive training work through discrete neuronal mechanisms, combined aerobic exercise and cognitive training might have a synergistic and superior effect on cognition compared to either intervention alone [22]. Traditionally, the combined effects of exercise and cognitive training have been investigated in a sequential manner, producing mixed results [6, 23-27]. It is thought that the heterogeneity between the interventions and the delivery of the interventions (i.e., sequentially) may be the primary factors for the inconsistencies $[28,29]$. A second interventional delivery option is simultaneous (dual-task) exercise + cognition training (i.e., "exergame"); however, there are limited studies that have investigated this delivery modality [29]. Anderson-Hanley [30] reported results from an exergame intervention (i.e., the "Cybercycle" Study) (cycling + virtual reality path finding and competition with a shadow cycler), a multi-site cluster RCT in which the cognitive benefit of cybercycling showed superior cognitive effects than traditional stationary cycling, for older adults living independently. More recently, Barcelos [28] compared cycling alone to virtual cycling with a concurrent video game task and found greater effects on cognitive measures from the cycling plus gaming intervention. However, similar studies have not been conducted in older adults with SCD.

\section{Study aims}

The purpose of this phase II RCT is to test the efficacy and additive/synergistic effects of an exergames intervention (simultaneous moderate intensity cycling and cognitive training), in comparison to cycling only and attention control (stretching) on cognition and aerobic fitness in older adults with SCD. The specific aims and hypotheses of the study are described as follows:

Aim 1: Determine the efficacy of the exergame in older adults with SCD.

Hypothesis: Exergame will improve global cognition more than cycling only and attention control.

Aim 2: Assess the distraction effect of an exergame on aerobic fitness.

Hypothesis 2a: Exergame participants will achieve similar gains in aerobic fitness to cycling participants. Hypothesis 2b. Exergame participants will achieve similar exercise intensity targets to cycling participants.

\section{Methods}

\section{Design}

The RCT will use a single-blind, 3-parallel group design, guided by CONSORT [31] and SPIRIT [32] guidelines $(n=96)$. We will use a 2:1:1 allocation ratio to randomize 48 subjects to exergame, 24 to cycling only, and 24 to attention control within age strata $(<75$ and $\geq$ 75 years) and using permuted blocks of 4 and 8 participants. Based on our previous studies, we anticipate screening approximately 288 individuals to enroll 96 participants. Cognition and aerobic fitness will be assessed at baseline and 3 months. This study was approved by the university Institutional Review Board and registered at ClinicalTrials.gov on 17 March 2020 (study identifier NCT04311736).

\section{Setting}

Screening and data collections will occur in a private room at the Clinical and Translational Science Institute or Laboratory of Clinical Physiology at the University of Minnesota, local Young Men's Christian Association (YMCA) gym, or senior center in close proximity to participant's residence. The interventions will occur at a local YMCA gym, senior center, or the participant's residence.

\section{Study population Recruitment}

We plan to enroll 96 participants with a proactive recruitment plan, including presentations at local YMCAs and senior living facility partners, exhibits at relevant 
conferences, mass email sends, advertisements through social media (i.e., Facebook and Instagram), and recruitment material distributions (i.e., fliers) in the communities.

\section{Eligibility and screening}

Potential participants who respond to our recruitment strategies and initiate contact with us will be carefully screened for eligibility and exercise safety using a threestep procedure spread over the course of 2-4 weeks, including a phone interview, an in-person interview, and a shuttle walk test (SWT) to ensure they meet our eligibility criteria (Table 1$)$.

1. Phone interview: The staff will receive the respondent's verbal consent for participating in a 20-30-min phone interview. The phone interview will evaluate the presence of SCD, health history, contraindications to exercise [33], and cognitive status (telephone interview for cognitive status (TICS)) [34]. Potential participants who are not excluded based on the phone screen will be scheduled for an in-person interview.

2. In-person interview: Informed consent, Health Insurance Portability and Accountability Act (HIPA A) authorization, and permission for release of medical records will be obtained by study staff. During informed consent, individuals will be informed about the study procedures, compensation, time commitment, potential risks and benefits, compensations, data collection, the voluntary nature of study participation, and contact within and outside the study team for human subjects. The study staff will corroborate phone screen data; administer questionnaires including Geriatric Depression Scale (GDS) [35], Beck's Anxiety Inventory (BAI) [36], and Geriatric Anxiety Scale (GAS-10) [37]; and conduct a focused physical assessment. The study coordinator will summarize screening data for review by the investigators to determine eligibility. For eligible participants, letters will be sent to the potential participant's medical providers to obtain medical clearance for exercise and for excluding the contribution of psychological symptoms to SCD.

3. Shuttle walk test (SWT): Upon receiving exercise safety clearance, participants will complete a SWT to further rule out clinical signs and symptoms indicative of symptomatic cardiovascular disease and to obtain peak heart rate (HR) to be used for purposes of exercise prescription. Resting seated and standing HR and blood pressure will be evaluated to ensure safety prior to performing the test. HR will be continuously monitored during exercise. The test will be stopped if any clinical signs or symptoms develop [33]. HR and blood pressure will be monitored for $6 \mathrm{~min}$ following completion of the SWT to ensure residual stability. Those who successfully complete the SWT (i.e., develop no signs and symptoms of symptomatic cardiovascular disease) will be formally enrolled in the study upon completing baseline data collection and subsequently randomized.

\section{Sample size and power}

Enrollment of 96 subjects with $12 \%$ attrition at 3 months will give us $80 \%$ power to detect a .85 effect size between exergame and attention control.

\section{Variables and their measures Primary and secondary outcomes}

The primary outcome (Table 2) for this RCT is episodic memory, while secondary outcomes include executive function, global cognition, and aerobic fitness. Outcomes will be assessed at baseline and at 3 months by data collectors who are blinded to group allocation and previously collected data. All cognitive outcomes will be assessed through the NIH Toolbox Cognitive Battery (NIHTB-CB) [38, 39] according to NIH Toolbox Assessment Center instructions [39]. The NIHTB-CB, an interactive personal application device (iPAD) based battery of executive function, attention, episodic memory, language, processing speed, and working memory tests, was developed within the NIH Blueprint for Neuroscience Research and has been validated and normed in a broad sample of the US population [38, 39]. This battery yields

Table 1 Eligibility criteria

\section{Exclusionary criteria}

a) Resting heart rate $>100$ or $<50$ beats/min with symptoms

b) Dementia or mild cognitive impairment (self-report, diagnosis, or scoring $<26$ on the TICS

c) Neurological or major psychiatric disorder likely causing SCD

d) Alcohol or chemical dependency that likely is causing SCD

e) Current enrollment in another intervention study

f) ACSM contraindications to exercise or other factors that make exercise impossible or unsafe

g) Inability to read due to illiteracy or poor eyesight 
Table 2 Data collected from participants during the study

\begin{tabular}{|c|c|c|c|c|}
\hline Variables & Measures (data type) & Data collection type & $\begin{array}{l}\text { Screening } \\
\text { period }\end{array}$ & $\begin{array}{l}\text { Baseline/follow- } \\
\text { up period }\end{array}$ \\
\hline \multicolumn{5}{|l|}{ Cognitive outcomes } \\
\hline Episodic memory & $\begin{array}{l}\text { Picture Sequence Memory Test, Auditory Verbal } \\
\text { Learning Test (both continuous) }\end{array}$ & $\mathrm{NIH}$ toolbox (iPAD) & & $x$ \\
\hline Executive function & $\begin{array}{l}\text { Cognitive Battery Flanker Inhibitory Control, } \\
\text { Attention Test and Dimensional Change Card } \\
\text { Sort Test (both continuous) }\end{array}$ & NIH toolbox (iPAD) & & $x$ \\
\hline Global cognition & $\begin{array}{l}\text { Composite score from all tests for discrete } \\
\text { cognitive domains }\end{array}$ & NIH toolbox (iPAD) & & $x$ \\
\hline \multicolumn{5}{|l|}{ Aerobic fitness outcomes } \\
\hline Aerobic fitness & SWT (continuous) & Performance-based test & & $x$ \\
\hline \multicolumn{5}{|l|}{ Other variables } \\
\hline Depression & GDS (continuous) & & $x$ & \\
\hline Anxiety & (Gas-10), BAI (both continuous) & & $x$ & \\
\hline Physical function & SPPB, 6MWT (both continuous) & Performance-based test & & $x$ \\
\hline Medical change & $\begin{array}{l}\text { Medical diagnoses, falls, medications } \\
\text { (all categorical) }\end{array}$ & & Monthly & \\
\hline Demographics & $\begin{array}{l}\text { Age, education (both continuous) sex, } \\
\text { race (both discrete) }\end{array}$ & Self-report, paper form & $x$ & \\
\hline Physical activity & $\begin{array}{l}\text { Accelerometry, PASE questionnaire } \\
\text { (both continuous) }\end{array}$ & $\begin{array}{l}\text { Wrist device, pencil-paper- } \\
\text { based questionnaire }\end{array}$ & Monthly & \\
\hline Intervention adherence & Percent (continuous) & REDcap report & & \\
\hline $\begin{array}{l}\text { Usability, acceptability, and } \\
\text { satisfaction of exergame }\end{array}$ & Likert scale (ordinal) & $\begin{array}{l}\text { Pencil-paper-based } \\
\text { questionnaire }\end{array}$ & Monthly & \\
\hline
\end{tabular}

6MWT 6-min walk test, BAI Beck's Anxiety Inventory, Gas-10 Geriatric Anxiety Scale - 10 item, GDS Geriatric Depression Scale, PASE Physical Activity Scale for the Elderly, SWT shuttle walk test

summary scores such as the Cognitive Functioning Composite Score, in addition to individual measure scores as outlined below [38, 39].

\section{Episodic memory}

Episodic memory refers to cognitive processes involved in the acquisition, storage, and retrieval of new information and will be measured using the Picture Sequence Memory Test (PSMT) [39] and Auditory Verbal Learning Test (Rey) (RAVLT) [39] (supplementary measure). The PSMT has excellent test-retest reliability $(r=0.84)$ and is strongly correlated with the RAVLT, $(r=.64)$, reflecting good convergent validity [40].

\section{Executive function}

Executive function refers to the capacity to plan, organize, and monitor the execution of goal-directed behaviors such as set-shifting and inhibitory control and will be measured by the Flanker Inhibitory Control and Attention Test [39] and Dimensional Change Card Sort Test [39]. Both tests demonstrate excellent sensitivity to age-related changes during adulthood, excellent test-retest reliability $(r=.85)$, and adequate to good convergent validity $(r=.52-.55)[41]$.

\section{Global cognition}

Global cognition refers to the overall cognitive ability and will be calculated as the NIHTB-CB Cognitive Functioning Composite Score (i.e., total score). The Cognitive Functioning Composite Score is calculated from the following domain-specific scores: language (Picture Vocabulary Test and Oral Reading Recognition Test), attention (Flanker Inhibitory Control and Attention Test), processing speed (Pattern Comparison Processing Speed Test and Oral Symbol Digit Test [supplementary measure]), and working memory (List Sorting Working Memory Test) in addition to the tests used to measure episodic memory and executive function. The composite score has high psychometric measurements including internal consistency (Cronbach's alpha $=.77$ ), test-retest reliability $(r=.90)$, and convergent validity $(r=.89)$ in adults [42].

\section{Aerobic fitness}

Aerobic fitness will be assessed using the SWT, following the guidelines by Singh and colleagues [43]. Participants will be required to walk along a level, 10-m course at a previously determined speed dictated by signals from an audio recording. Laps will be counted for calculation of peak walking distance achieved during the 
SWT. The test will be considered finished when the participant is not able to maintain the required speed (more than $0.5 \mathrm{~m}$ from the cone) or for some other reported symptom that warrants test termination (i.e., American College of Sports Medicine [ACSM] clinical sign or symptom for exercise test termination) [33]. Because older adults with SCD may have trouble multitasking and remembering test instructions, we will provide additional instructions to ensure proper testing methodology including by providing additional verbal instruction including (1) "stop" when participants arrive at the marker before the beep, (2) "go" when the single beep for walking sounds, and (3) "go and walk faster now" when the triple beep sounds, indicating faster pace required [44]. The SWT reliability is well established in older adults with multiple chronic conditions (interclass correlation 0.91-.97) [45, 46]. Additionally, the peak walking distance on SWT is highly correlated to peak oxygen consumption derived from cardiopulmonary exercise testing in older adults with multiple chronic conditions [47], making it a valid test for assessing aerobic fitness.

\section{Study procedure}

\section{Study preparation and randomization}

All staff will be adequately trained to ensure participant safety, blinding, data quality, and protocol adherence based on the Study Manual of Operations. Prior to any recruitment, the statistician will create a randomization schedule in Research Electronic Data Capture (REDCap). Qualified participants will be randomized after completing baseline data collection. The study coordinator will $\log$ into the REDCap randomization module and enter a participant's name/age. The randomization module will assign the participant to a group and record the assignment in a database that is only accessible to unblinded staff.

\section{Data collection}

Enrolled subjects will be scheduled to complete baseline data collection. Subsequent data collection will occur at 3 months. Data collectors will be independent from study interventionists to help insure data integrity. To ensure the blindness of the data collectors from the intervention assignment, the data collectors will not interact with enrolled subjects except for data collection.

\section{Delivery of the assigned activity for each group}

Within 2 weeks of completing baseline data collection, participants will start their assigned activity: exergames, cycling only, or attention control. Each activity will consist of a total of three weekly, supervised sessions for 3 months (36 total sessions). One interventionist will supervise up to three participants in a single visit (1:3 interventionist/participant ratio) in the same activity. The sessions will be delivered over up to a 14-week period to account for missing sessions due to vacations and illnesses.

\section{Cycling group}

Subjects will cycle on recumbent stationary cycles at moderate intensity individualized as $50-70 \%$ of heart rate reserve (HRR) and/or 11-14 on Borg Category Ratio-15 Rating of Perceived Exertion (RPE) Scale. HRR will be calculated by subtracting resting HR (after 10min quiet resting) from the peak $H R$ achieved during the SWT. Cycling will be progressed from $50-60 \%$ of HRR or RPE $11-12$ for $30-35$ min in session 1 and will be alternatively increased by $5 \%$ of HRR (or 1-point on Borg) or 5 -min increments as tolerated up to $60-70 \%$ of HRR (or RPE 12-14) for 50 min a session over time. All sessions will include a 5-min warm-up and 5 cool-down before and after cycling, in addition to the prescribed exercise duration, following the ACSM guidelines [33]. In each session, the interventionists will help subjects put on a Polar ${ }^{\text {rm }}$ wireless HR monitor (RS400, Lake Success, $\mathrm{NY}$ ), take resting HR and blood pressure, and monitor HR, RPE, talk test, and signs and symptoms every $5 \mathrm{~min}$, and blood pressure every $15 \mathrm{~min}$.

\section{Exergame}

Subjects will cycle as described in the cycling protocol while engaging in cognitive training for the duration of cycling. Cognitive training will include one level of difficulty with six task scenarios in the context of three virtual worlds (environments): "Small-Town Downtown, " "Underwater World," and "Wild West." For example, the virtual world of "Small-Town Downtown" will be composed of shops, restaurants, storefronts, and office buildings. Each environment will be open-world and static and will not change over time or between game sessions. This will allow the participant to memorize the layout over multiple play sessions. Visual cues ("Landmarks") will serve as memorization guides for navigation. Landmark examples for "Small-Town Downtown" include unique buildings, bridges, statues, parks, and other true-to-the-environment objects. Scenarios will have high ecological validity. Scenario examples in "Small-Town Downtown" include (1) picking up and dropping off a delivery for a friend, (2) visiting the post office to mail a letter, (3) sorting books at the library, and (4) going shopping at the grocery store. In each of the environments, the participant must follow directions and navigate to a destination where the cognitive task will be performed. For each scenario, participant start position within the environment will be randomly assigned by the system. A scenario-specific cognitive task will be triggered. The game will inform the 
participant of the assigned task and, automatically, determine and inform the participant of the fastest route from the starting point to the scenario destination where the task will be performed. The participant will be required to remember the assigned task, follow the directions and navigate to the destination, and then remember what action is required to complete the task. The participant will move forward through the environment by pedaling the stationary cycle. Left and right turns along the route will be input by the participant through buttons on the game controller. To enable a smooth transition into the cognitive task while maintaining cycling movement, the game's camera will continue to progress in a forward direction to match participant pedaling speed, while the level environment fades into a desaturated surreal version of each scenario's setting.

\section{Attention control}

Participants will participate in passive stretching that we have previously tested $[48,49]$ and that are matched to session durations for cycling and exergame groups. Light intensity (RPE $\leq 9$ or $\mathrm{HRR} \leq 30 \%$ ) stretching exercises include seated movements and static stretches that induce no changes in aerobic fitness.

\section{Treatment fidelity}

This study was designed to ensure treatment fidelity based on the NIH Behavior Change Consortium recommendations [50] and our experiences [48, 49]. The investigators and the study coordinator will oversee adherence to the protocol by all staff through review focused on screening compliance, study outcomes, intervention compliance (i.e., percent of exercise sessions attended, percentage of session duration in target intensity range, and exercise dose), and adverse events. Investigators and the study coordinator will ensure data creation and completeness through quality check and audits. Weekly meetings will be held among the investigators and study staff to ensure compliance to study protocol, while monthly fidelity checks will be performed (total $3 \%$ of intervention sessions) by the investigators to ensure accurate implementation of intervention protocols by using a previously used Fidelity Checklist [48]. Staff will be retrained as needed. The investigators will review $3 \%$ of session case forms (i.e., exercise logs) to assess for protocol drift and completeness.

\section{Safety, retention, adherence, and validation}

We have built in many strategies that have been successfully utilized in our preliminary studies to protect participants against risks, including ongoing monitoring of health status changes, appropriate equipment and personnel training, individualized prescriptions and supervised delivery for cycling and exergames training, and careful screening and informed consent. Interventionists will be fully trained using the Study Manual of Operations and will be able to consult with the investigators on an immediate, as-needed basis as well as at weekly meetings. During the course of the intervention, if a participant develops a contraindication to exercise, interventions will be terminated until the participant is recleared to resume exercise by their primary care provider or cardiologist.

\section{Data management}

\section{Data entry, coding, and storage}

All participant data will be identified by a screening identification (ID) and a study ID for enrolled participants. Access to the links between name and IDs will be restricted to the study coordinator and interventionists involved in screening. Data collection forms returned to the research office by the study staff will be reviewed by the study coordinator for accuracy and completeness before entry into the study REDCap database [51, 52]. Data collection forms will then be stored in a specific office that is further locked and contains a lockable storage cabinet specific for data collection storage. Cognitive data from the NIH Toolbox Cognitive Battery will be exported from study iPAD into a university email account and then subsequently uploaded into REDCap by data collectors. Additionally, the study coordinator will verify data accuracy and completeness in REDCap. Any missing data will be assigned to a data collector for collection. If a participant withdraws from the study, all attempts will be made to collect data to allow for inclusion in the analysis. Reasons for withdrawal will be recorded. Data audits of electronic outcome data will be conducted by the study coordinator. If any discrepancies in scoring between the study staff who collected data and the study staff who entered data, a meeting with the investigators or study coordinator and involved study staff will be undertaken to ensure the correct data is in REDCap.

\section{Statistical analyses}

All variables will be summarized using appropriate descriptive statistics (e.g., means/standard deviation [SD] for continuous measures, and frequencies for categorical variables). Distributions will be visually inspected for outliers, and determine the best link and distribution combination for each outcome using the quasi information criteria [53]. Statistical assumptions will be checked. Baseline variables will be compared among the 3 groups to determine systematic differences using Pearson's chisquare test (categorical variables) and one-way analysis of variance (ANOVA) or Kruskal-Wallis (continuous variables), as appropriate. Analysis will be accomplished using Statistical Product and Service Solutions (SPSS) 
for Windows or Statistical Analysis System (SAS). Variables that differ significantly among groups will be included as covariates in the models described below. All analyses will follow the intention-to-treat principle.

Aim 1: Determine the efficacy of the exergame in older adults with SCD. To test our hypothesis that exergame will improve global cognition more than cycling only and attention control, the ANOVA will be performed. If statistically significant results are identified, then we will perform post hoc analysis to determine how the changes in cognition differ between groups. If homogeneity of variances are supported across the three groups, we will conduct Tukey's honestly significant different post hoc test. If homogeneity of variances are violated, then we will perform the Games Howell post hoc test. If significant covariates are identified, then analysis of covariance (ANCOVA) will be used instead. Linear simple and multiple regressions will be performed to assess association between the effects of exergames on global cognition and aerobic fitness. A $p$ value $<0.05$ will be used to determine statistical significance. Effect size (EF) calculated as $\mathrm{EF}=$ (mean exergames group - mean stretching group)/SD (stretching group) will be used to assess the clinical significance of the effects of exergames on cognitive and aerobic fitness outcomes listed above. Aim 2: Assess the distraction effect of exergame on aerobic fitness. Our hypothesis that exergame participants will achieve similar gains in aerobic fitness to cycling participants will be analyzed similarly to aim 1 hypothesis using ANOVA and post hoc test. In addition, heart rate monitor data will be utilized to compute intensity dose, to compare objective effort given during exergame and cycling only sessions. We hypothesize that the average \%HRR achieved by exergame participants will not be significantly different compared to cycling only participants. A standard formula will be used to quantify the intensity dose and is as follows. Percent HRR (\%HRR) averaged in each session will be calculated from the following variables (EHR = exercise session average heart rate, PHR = peak heart rate from SWT, and RHR = resting heart rate from SWT $)$ and formula: \%HRR = [(EHR - RHR $)] /$ (PHR - RHR)]. The \%HRR will be averaged across all sessions to give a quantifiable index of intensity to be used as a surrogate measure of distraction. Differences between exergames and cycling only groups on average $\%$ HRR will be assessed by independent $T$ test.

\section{Ethics}

This study was approved by the university Institutional Review Board (IRB), and any amendments or subsequent changes or to the protocol will be submitted to the IRB for approval. Written consent takes place during the inperson interview. Participant's capacity to consent will be assessed by staff through use of the UCSD Brief Assessment of Capacity to Consent form (UBACC) [54] modified for exergame. A participant must score a "2" on items 1, 2, 4, 6, 7, and 9 on the assessment for inclusion in the study. If a participant scores less than 2 on any of these items, the staff will re-explain the study, and then ask the participant to return on another day to retake the UBACC. If the participant scores $<2$ on any of these items again, then the subject is not eligible to participate in the study. Consenting will be an ongoing process during the study as verbal consent will be obtained at the beginning of each activity session. Participation in exercise is known to cause musculoskeletal pain or discomfort during exercise or $24-48 \mathrm{~h}$ following exercise (due to commonly seen delayed onset muscle soreness). Study-related, anticipated adverse events such as these will not be reported to IRB; however, all studyrelated unanticipated adverse events (such as cardiopulmonary events) will be reported to the IRB and funding agency within 5 business days of the event.

\section{Dissemination}

Findings from the RCT will be disseminated through presentations and publications. Participants and their family members will receive a copy of the published main findings. All manuscripts will be authored by the study team and authorship will follow the established publication guidelines such as those of the International Committee of Medical Journal Editors. Access to the final trial data set will follow the guidelines of the National Institute on Aging.

\section{Discussion}

Developing interventions, with strong potential to prevent or delay the onset of $A D$ in persons with $S C D$ is critical. Aerobic exercise and cognitive training represent two promising interventions; however, their synergistic effects on preventing AD have not been evaluated. Our study will develop a novel, apple-TV, and iPad-based cognitive training that is immersive, naturalistic, and similar in tasks to real life, and therefore increases the ecologic validity of the treatment [29]. It is cognitively challenging but also increases exercise enjoyment.

All attempts have been undertaken to ensure the highest quality of study design and delivery of the Exergames Study. First, RCTs are considered the gold standard design for determining the causal effect of an intervention on an outcome. Alternatively, intervention trials commonly employ usual care or waitlist control groups or cross-over design. Usual-care or waitlist controls preclude assessing the Hawthorne or placebo effect of exergames, while cross-over designs are ideal for 
interventions without carry-over effects, which is also not the case for exergames. In addition, we have built in four strategies to prevent unblinding, another potential problem occurring in RCTs. The four unblinding prevention strategies include (1) assessors will not interact with other staff who are not blinded (i.e., exercise interventionists) and will participate in separate meetings, (2) investigators will be blinded to group assignments, (3) participants will be blinded to the study aims and reminded as needed not to discuss their experiences with the outcome assessor, and (4) randomization will be completed using permuted blocks. Additionally, age is a known, strong factor affecting cognition. While our aims are not focused on age, we will stratify enrollment by age to equalize age distribution across groups $[55,56]$.

In summary, the Exergames Study will be the first RCT to examine the additive/synergistic effects of a novel simultaneous moderate intensity, supervised aerobic exercise + cognitive training intervention on cognition and aerobic fitness. This study looks to advance AD prevention research by providing precise immediate and long-term effect-size estimates of an aerobic exercise intervention delivered with simultaneous cognitive training to inform future fully powered, large scale phase III RCTs.

\section{Trial status}

ClinicalTrials.gov identifier NCT04011267, version 1.0, 17 March 2020. Registered on 17 March 2020 (reterospectively registered) and at the time of manuscript submission has not been updated. The status of the trial at the time of manuscript submission is open for enrollment. Participant enrollment began on 27 December 2019 and we expect enrollment accrual to complete in 2022.

\section{Abbreviations}

ACSM: American College of Sports Medicine; AD: Alzheimer's disease; ANCOVA: Analysis of covariance; BAl: Beck's Anxiety Inventory; CONSORT: Consolidated Standards of Reporting Trials; EF: Effect size; Gas10: Geriatric Anxiety Scale - 10-item version; HIPPA: Health Insurance Portability and Accountability Act; HR: Heart rate; HRR: Heart rate reserve; ID: Identification; iPAD: Interactive personal application device; IRB: Institutional review board; MCl: Mild cognitive impairment; NIHTBCB: NIH Toolbox Cognitive Battery; PSMT: Picture Sequence Memory Test; RAVLT: Auditory Verbal Learning Test (Rey); RCT: Randomized controlled trial; REDcap: Research Electronic Data Capture; RPE: Rating of perceived exertion; SAS: Statistical Analysis System; SCD: Subjective cognitive decline; SPIR IT: Standard Protocol Items: Recommendations for Interventional Trials; SPSS: Statistical Product and Service Solutions; SWT: Shuttle walk test; UBACC: USCD Brief Assessment of Capacity to Consent; VRCT: Virtual reality cognitive training; YMCA: Young Men's Christian Association

\section{Acknowledgements}

The CTSI was supported by the National Institutes of Health National Center for Advancing Translational Sciences of the National Institutes of Health Award Number UL1TR000114. The content is solely the responsibility of the authors and does not necessarily represent the official views of the National Institutes of Health. We appreciate the efforts of the study staff.

\section{Authors' contributions}

DS, FY, and TP designed the study. DS and FY supervise all aspects of the study implementation and drafted the manuscript. DS designed and supervises all aspects of the study related to exercise. TP designed the Exergame. All authors read and approved the final manuscript.

\section{Funding}

This study was funded by the Small Business Innovation Research from the National Institute on Aging of the National Institutes of Health (4R44AG055176-02). The funding body had no role in the design of the study and collection, analysis, interpretation of data, or in writing the manuscript.

\section{Availability of data and materials \\ Not applicable}

Ethics approval and consent to participate

This trial was approved by the university's IRB (MOD00000770). It was registered at ClinicalTrials.gov (a service of the US National Institutes of Health) identifier NCT04311736 (on 17 March 2020) under the name "Concurrent Aerobic Exercise and Cognitive Training to Prevent Alzheimer's in At-risk Older Adults (Exergames)." The consent process is outlined in the manuscript and informed consent will be obtained from all study participants. Any changes made to the protocol will be amended and communicated to the university IRB. The changes will also be included in the clinical trial register (https://clinicaltrials.gov/). This trial does not involve collecting biological specimens for storage.

\section{Consent for publication \\ Not applicable}

\section{Competing interests}

The authors declare that they have no competing interests.

\section{Author details}

'University of Minnesota School of Nursing, 5-160 WDH 1331, 308 Harvard St SE, Minneapolis, MN 55455, USA. ${ }^{2}$ Moai Technologies LLC, Minneapolis, MN, USA. ${ }^{3}$ Arizona State University, Health North Suite 301, 550 North 3rd Street, Mail Code 3020, Phoenix, AZ 85004, USA.

Received: 22 June 2020 Accepted: 7 December 2020

Published online: 06 January 2021

\section{References}

1. Jessen F, Amariglio RE, van Boxtel M, Breteler M, Ceccaldi M, Chetelat G, et al. A conceptual framework for research on subjective cognitive decline in preclinical Alzheimer's disease. Alzheimers Dement. 2014;10(6):844-52.

2. Sperling RA, Aisen PS, Beckett LA, Bennett DA, Craft S, Fagan AM, et al. Toward defining the preclinical stages of Alzheimer's disease: recommendations from the National Institute on Aging-Alzheimer's Association workgroups on diagnostic guidelines for Alzheimer's disease. Alzheimers Dement. 2011;7(3):280-92.

3. Cummings $\mathrm{J}$, Morstorf T, Zhong K. Alzheimer's disease drug-development pipeline: few candidates, frequent failures. Alzheimers Res Ther. 2014;6(4):37. https://doi.org/10.1186/alzrt269.

4. Coyle H, Traynor V, Solowij N. Computerized and virtual reality cognitive training for individuals at high risk of cognitive decline: systematic review of the literature. Am J Geriatr Psychiatry. 2015;23(4):335-59.

5. Davenport MH, Hogan DB, Eskes GA, Longman RS, Poulin MJ. Cerebrovascular reserve: the link between fitness and cognitive function? Exerc Sport Sci Rev. 2012:40(3):153-8.

6. Fissler P, Kuster O, Schlee W, Kolassa IT. Novelty interventions to enhance broad cognitive abilities and prevent dementia: synergistic approaches for the facilitation of positive plastic change. Prog Brain Res. 2013;207:403-34.

7. Angevaren M, Aufdemkampe G, Verhaar HJ, Aleman A, Vanhees L. Physical activity and enhanced fitness to improve cognitive function in older people without known cognitive impairment. Cochrane Database Syst Rev. 2008;(3): Cd005381. https://doi.org/10.1002/14651858.CD005381.pub3.

8. Fratiglioni L, Paillard-Borg S, Winblad B. An active and socially integrated lifestyle in late life might protect against dementia. Lancet Neurol. 2004;3(6):343-53. 
9. Laurin D, Verreault R, Lindsay J, MacPherson K, Rockwood K. Physical activity and risk of cognitive impairment and dementia in elderly persons. Arch Neurol. 2001;58(3):498-504.

10. Colcombe $\mathrm{S}$, Kramer AF. Fitness effects on the cognitive function of older adults: a meta-analytic study. Psychol Sci. 2003;14(2):125-30.

11. Lautenschlager NT, Cox KL, Flicker L, Foster JK, van Bockxmeer FM, Xiao J, et al. Effect of physical activity on cognitive function in older adults at risk for Alzheimer disease: a randomized trial. JAMA. 2008;300(9):1027-37.

12. Ohman H, Savikko N, Strandberg TE, Kautiainen H, Raivio MM, Laakkonen ML, et al. Effects of exercise on cognition: the Finnish Alzheimer disease exercise trial: a randomized, controlled trial. J Am Geriatr Soc. 2016;64(4):731-8.

13. Panza GA, Taylor BA, MacDonald HV, Johnson BT, Zaleski AL, Livingston J, et al. Can exercise improve cognitive symptoms of Alzheimer's disease? J Am Geriatr Soc. 2018;66(3):487-95.

14. Heyn P, Abreu BC, Ottenbacher KJ. The effects of exercise training on elderly persons with cognitive impairment and dementia: a meta-analysis. Arch Phys Med Rehabil. 2004:85(10):1694-704

15. Smith PJ, Blumenthal JA, Hoffman BM, Cooper H, Strauman TA, WelshBohmer K, et al. Aerobic exercise and neurocognitive performance: a metaanalytic review of randomized controlled trials. Psychosom Med. 2010;72(3): 239-52.

16. Martin M, Clare L, Altgassen AM, Cameron MH, Zehnder F. Cognition-based interventions for healthy older people and people with mild cognitive impairment. Cochrane Database Syst Rev. 2011;(1):Cd006220. https://doi.org/ 10.1002/14651858.CD006220.pub2.

17. Kueider AM, Parisi JM, Gross AL, Rebok GW. Computerized cognitive training with older adults: a systematic review. PLoS One. 2012;7(7):e40588.

18. Lovden M, Backman L, Lindenberger U, Schaefer S, Schmiedek F. A theoretical framework for the study of adult cognitive plasticity. Psychol Bull. 2010;136(4):659-76.

19. Rebok GW, Ball K, Guey LT, Jones RN, Kim HY, King JW, et al. Ten-year effects of the advanced cognitive training for independent and vital elderly cognitive training trial on cognition and everyday functioning in older adults. J Am Geriatr Soc. 2014;62(1):16-24.

20. Wolinsky FD, Vander Weg MW, Howren MB, Jones MP, Dotson MM. A randomized controlled trial of cognitive training using a visual speed of processing intervention in middle aged and older adults. PLoS One. 2013; 8(5):e61624.

21. Ball $K, E d w a r d s ~ J D$, Ross $L A$. The impact of speed of processing training on cognitive and everyday functions. J Gerontol B Psychol Sci Soc Sci. 2007;62 Spec No 1:19-31.

22. Suo C, Singh MF, Gates N, Wen W, Sachdev P, Brodaty $H$, et al. Therapeutically relevant structural and functional mechanisms triggered by physical and cognitive exercise. Mol Psychiatry. 2016;21(11):1633-42.

23. Fabre C, Chamari K, Mucci P, Masse-Biron J, Prefaut C. Improvement of cognitive function by mental and/or individualized aerobic training in healthy elderly subjects. Int J Sports Med. 2002;23(6):415-21.

24. Linde K, Alfermann D. Single versus combined cognitive and physical activity effects on fluid cognitive abilities of healthy older adults: a 4-month randomized controlled trial with follow-up. J Aging Phys Act. 2014;22(3):302-13.

25. Rahe J, Petrelli A, Kaesberg S, Fink GR, Kessler J, Kalbe E. Effects of cognitive training with additional physical activity compared to pure cognitive training in healthy older adults. Clin Interv Aging. 2015;10:297-310.

26. Oswald WD, Gunzelmann T, Rupprecht R, Hagen B. Differential effects of single versus combined cognitive and physical training with older adults: the SimA study in a 5-year perspective. Eur J Ageing. 2006;3(4):179.

27. Fiatarone Singh MA, Gates N, Saigal N, Wilson GC, Meiklejohn J, Brodaty H, et al. The Study of Mental and Resistance Training (SMART) study-resistance training and/or cognitive training in mild cognitive impairment: a randomized, double-blind, double-sham controlled trial. J Am Med Dir Assoc. 2014;15(12):873-80.

28. Barcelos N, Shah N, Cohen K, Hogan MJ, Mulkerrin E, Arciero PJ, et al. Aerobic and cognitive exercise (ACE) pilot study for older adults: executive function improves with cognitive challenge while exergaming. J Int Neuropsychol Soc. 2015;21(10):768-79.

29. Tait JL, Duckham RL, Milte CM, Main LC, Daly RM. Influence of sequential vs. simultaneous dual-task exercise training on cognitive function in older adults. Front Aging Neurosci. 2017;9:368.

30. Anderson-Hanley C, Arciero PJ, Brickman AM, Nimon JP, Okuma N, Westen SC, et al. Exergaming and older adult cognition: a cluster randomized clinical trial. Am J Prev Med. 2012;42(2):109-19.
31. Schulz KF, Altman DG, Moher D. CONSORT 2010 statement: updated guidelines for reporting parallel group randomized trials. Ann Intern Med. 2010;152(11):726-32.

32. Chan AW, Tetzlaff JM, Altman DG, Laupacis A, Gotzsche PC, Krleza-Jeric K, et al. SPIRIT 2013 statement: defining standard protocol items for clinical trials. Ann Intern Med. 2013;158(3):200-7.

33. American College of Sports Medicine. In: Riebe D, Ehrman JK, Liguori G, editors. ACSM's Guidelines for Exercise Testing and Prescription. 10th ed. Philadelphia: Wolters Kluwer; 2018.

34. Seo EH, Lee DY, Kim SG, Kim KW, Kim DH, Kim BJ, et al. Validity of the telephone interview for cognitive status (TICS) and modified TICS (TICSm) for mild cognitive imparment $(\mathrm{MCl})$ and dementia screening. Arch Gerontol Geriatr. 2011:52(1):e26-30.

35. McDowell I, Newell C. Measuring health: a guide to rating scales and questionnaires. 2nd ed. New York: Oxford University Press; 1996.

36. Beck AT, Epstein N, Brown G, Steer RA. An inventory for measuring clinical anxiety: psychometric properties. J Consult Clin Psychol. 1988;56(6):893-7.

37. Mueller AE, Segal DL, Gavett B, Marty MA, Yochim B, June A, et al. Geriatric Anxiety Scale: item response theory analysis, differential item functioning, and creation of a ten-item short form (GAS-10). Int Psychogeriatr. 2015;27(7):1099-111.

38. Gershon RC, Wagster MV, Hendrie HC, Fox NA, Cook KF, Nowinski CJ. NIH toolbox for assessment of neurological and behavioral function. Neurology. 2013;80(11 Suppl 3):S2-6.

39. Gershon R, Wagster M. Summary of NIH Toolbox: National Institutes of Health and Northwestern University; 2012. Available from: http://www. nihtoolbox.org/Resources/Tools\%20for\%20authors\%20researchers/Pages/ default.aspx.

40. Dikmen SS, Bauer PJ, Weintraub S, Mungas D, Slotkin J, Beaumont JL, et al. Measuring episodic memory across the lifespan: NIH Toolbox Picture Sequence Memory Test. J Int Neuropsychol Soc. 2014;20(6):611-9.

41. Zelazo PD, Anderson JE, Richler J, Wallner-Allen K, Beaumont JL, Conway KP, et al. NIH Toolbox Cognition Battery (CB): validation of executive function measures in adults. J Int Neuropsychol Soc. 2014;20(6):620-9.

42. Heaton RK, Akshoomoff N, Tulsky D, Mungas D, Weintraub S, Dikmen S, et al. Reliability and validity of composite scores from the NIH Toolbox Cognition Battery in adults. J Int Neuropsychol Soc. 2014;20(6):588-98.

43. Singh SJ, Morgan MD, Scott S, Walters D, Hardman AE. Development of a shuttle walking test of disability in patients with chronic airways obstruction. Thorax. 1992:47(12):1019-24.

44. Salisbury DL, Yu F. Aerobic fitness and cognition changes after exercise training in Alzheimer's disease. J Clin Exerc Physiol. 2017;5(2):7.

45. Singh S, Moiz JA, Ali MS, Talwar D. Reliability, validity, and responsiveness of the incremental shuttle walk test in patients with interstitial lung disease. J Cardiopulm Rehabil Prev. 2018:38(6):425-9.

46. Wilkinson TJP, Xenophontos SM, Gould DWP, Vogt BPP, Viana JLP, Smith ACP, et al. Test-retest reliability, validation, and "minimal detectable change" scores for frequently reported tests of objective physical function in patients with non-dialysis chronic kidney disease. Physiother Theory Pract. 2019;35(6):565-76.

47. Parreira VF, Janaudis-Ferreira T, Evans RA, Mathur S, Goldstein RS, Brooks D. Measurement properties of the incremental shuttle walk test. A systematic review. Chest. 2014:145(6):1357-69.

48. Yu F, Bronas UG, Konety S, Nelson NW, Dysken M, Jack C Jr, et al. Effects of aerobic exercise on cognition and hippocampal volume in Alzheimer's disease: study protocol of a randomized controlled trial (the FIT-AD trial). Trials. 2014;15:394

49. Yu F, Lin FV, Salisbury DL, Shah KN, Chow L, Vock D, et al. Efficacy and mechanisms of combined aerobic exercise and cognitive training in mild cognitive impairment: study protocol of the ACT trial. Trials. 2018;19(1):700.

50. Bellg AJ, Borrelli B, Resnick B, Hecht J, Minicucci DS, Ory M, et al. Enhancing treatment fidelity in health behavior change studies: best practices and recommendations from the NIH Behavior Change Consortium. Health Psychol. 2004:23(5):443-51.

51. Harris PA, Taylor R, Thielke R, Payne J, Gonzalez N, Conde JG. Research electronic data capture (REDCap)--a metadata-driven methodology and workflow process for providing translational research informatics support. J Biomed Inform. 2009:42(2):377-81.

52. Harris PA, Taylor R, Minor BL, Elliott V, Fernandez M, O'Neal L, et al. The REDCap consortium: Building an international community of software platform partners. J Biomed Inform. 2019;95:103208. 
53. Taguri M, Matsuyama Y, Ohashi Y. Model selection criterion for causal parameters in structural mean models based on a quasi-likelihood. Biometrics. 2014;70(3):721-30.

54. Jeste DV, Palmer BW, Appelbaum PS, Golshan S, Glorioso D, Dunn LB, et al. A new brief instrument for assessing decisional capacity for clinical research. Arch Gen Psychiatry. 2007;64(8):966-74.

55. Lindsay J, Laurin D, Verreault R, Hebert R, Helliwell B, Hill GB, et al. Risk factors for Alzheimer's disease: a prospective analysis from the Canadian Study of Health and Aging. Am J Epidemiol. 2002;156(5):445-53.

56. Seshadri S, Wolf PA, Beiser A, Au R, McNulty K, White R, et al. Lifetime risk of dementia and Alzheimer's disease. The impact of mortality on risk estimates in the Framingham study. Neurology. 1997;49(6):1498-504.

\section{Publisher's Note}

Springer Nature remains neutral with regard to jurisdictional claims in published maps and institutional affiliations.

Ready to submit your research? Choose BMC and benefit from:

- fast, convenient online submission

- thorough peer review by experienced researchers in your field

- rapid publication on acceptance

- support for research data, including large and complex data types

- gold Open Access which fosters wider collaboration and increased citations

- maximum visibility for your research: over $100 \mathrm{M}$ website views per year

At BMC, research is always in progress.

Learn more biomedcentral.com/submissions 